\title{
A randomised controlled trial to assess the efficacy of Laparoscopic Uterosacral Nerve Ablation (LUNA) in the treatment of chronic pelvic pain: The trial protocol [ISRCTN4 I I 96 I 5 I] The LUNA Trial Collaboration*
}

Address: Department of Obstetrics and Gynaecology, Birmingham Clinical Trials Unit and Department of Public Health and Epidemiology, University of Birmingham B15 2TT, UK

Email: The LUNA Trial Collaboration* - pallavi.latthe@bwhct.nhs.uk

* Corresponding author

Published: 08 December 2003

BMC Women's Health 2003, 3:6
Received: 24 July 2003

Accepted: 08 December 2003

This article is available from: http://www.biomedcentral.com/l472-6874/3/6

(c) 2003 The LUNA Trial Collaboration; licensee BioMed Central Ltd. This is an Open Access article: verbatim copying and redistribution of this article are permitted in all media for any purpose, provided this notice is preserved along with the article's original URL.

\begin{abstract}
Background: Chronic pelvic pain is a common condition with a major impact on health-related quality of life, work productivity and health care utilisation. The cause of the pain is not always obvious as no pathology is seen in $40-60 \%$ of the cases. In the absence of pathology there is no established treatment. The Lee-Frankenhauser sensory nerve plexuses and parasympathetic ganglia in the uterosacral ligaments carry pain from the uterus, cervix and other pelvic structures. Interruption of these nerve trunks by laparoscopic uterosacral nerve ablation (LUNA) may alleviate pain. However, the balance of benefits and risks of this intervention have not been reliably assessed. LUNA has, nevertheless, been introduced into practice, although there remains controversy regarding indications for LUNA. Hence, there is an urgent need for a randomised controlled trial to confirm, or refute, any worthwhile effectiveness. The principal hypothesis is that, in women with chronic pelvic pain in whom diagnostic laparoscopy reveals either no pathology or mild endometriosis (AFS score $\leq 5$ ) LUNA alleviates pain and improves life quality at 12 months.
\end{abstract}

Methods/Design: The principal objective is to test the hypothesis that in women with chronic pelvic pain in whom diagnostic laparoscopy reveals either no pathology or mild endometriosis (AFS score $\leq 5)$ LUNA alleviates pain and improves life quality at 12 months. A multi-centre, prospective, randomised-controlled-trial will be carried out with blind assessment of outcomes in eligible consenting patients randomised at diagnostic laparoscopy to LUNA (experimental group) or to no pelvic denervation (control group). Postal questionnaires including visual analogue scale for pain (primary outcome), an index of sexual satisfaction and the EuroQoL 5D-EQ instrument (secondary outcomes) will be administered at 3,6 and I 2 months. The primary assessment of the effectiveness of LUNA will be from comparison of outcomes at the one-year follow-up, although the mediumterm and longer-term risks and benefits of LUNA will also be evaluated.

The sample size for this trial has been estimated as 420 patients in total using the hypothesis that LUNA will alleviate pain symptoms (i.e. reduce pain scores on a VAS) more than no intervention at one-year following diagnostic laparoscopy and taking into consideration $20 \%$ loss to follow-up. The intention to treat analysis to address the principal research questions will be conducted using the one-year follow-up data. 


\section{Background}

Pelvic pain remains the single most common indication for referral to a Gynaecology clinic accounting for $20 \%$ of all outpatient appointments $[1,2]$. Five percent of all new appointments are for chronic pelvic pain[3]. It continues to be one of the most difficult and perplexing problems encountered in Gynaecology. Pelvic pain has a major impact on health-related quality of life, work productivity and health care utilisation. It is also a major cause of workplace absenteeism [4]. An estimated 158 million pounds are spent annually on the management of this condition in the health service [5]. In primary care, the annual prevalence is $38 / 1000$ in women aged $15-73$, a rate comparable to that of asthma (37/1000) and chronic back pain (41/1000)[6].

An initial troublesome problem in addressing research issues related to pelvic pain is the difficulty in making a definite diagnosis. The following definition is commonly used and will be adopted for our research proposal: Constant or intermittent, cyclic or acyclic pain, that persists for 6 months or more and includes dysmenorrhoea, deep dyspareunia and intermenstrual pain [7].

An effective treatment for pelvic pain has evaded gynaecologists for centuries. Even today only 20-25\% patients respond to conservative management [8]. When such treatment fails, a diagnostic laparoscopy is usually performed $[2,3,9]$. Chronic pelvic pain accounts for up to $40-60 \%$ of all diagnostic laparoscopies [2]. The cause of the pain is not always obvious as no pathology is seen in $40-60 \%$ of the cases. When definite pathology is found the likely causes are endometriosis (25\%) and adhesions (25\%) [2]. There is evidence that negative findings at laparoscopy and follow up with ultrasound will provide reassurance and relief to some patients. For patients without obvious pathology there is no established treatment.

In recent years, operative laparoscopy has developed rapidly and now makes laparoscopic pelvic denervation a practicable option in the management of chronic pelvic pain. The nerve plexuses and parasympathetic ganglia in the uterosacral ligaments, first described in the last century [10], and subsequently confirmed [11,12], carry "pain" from the uterus, cervix and other pelvic structures. The surgical approach to the management of pelvic pain is based on interruption of these nerve trunks that can be achieved by transection of uterosacral ligaments.

The transection of the uterosacral ligaments and the nerve plexuses it contains is the simplest of the surgical procedures for pelvic pain. The original work by Doyle described vaginal and abdominal approaches to divide the attachments of the uterosacral ligaments to the cervix $[13,14]$. With the wider use of minimal access therapy there is a renewed interest in the division of the Frankenhauser nerve plexus in the uterosacral ligaments laparoscopically using lasers or electro-diathermy. In an attempt to relieve patients' symptoms clinicians frequently perform laparoscopic uterosacral nerve ablation, or LUNA as it is sometimes called. However the efficacy of this procedure has not been assessed objectively using methodologically sound research.

\section{The Need for the LUNA Trial}

Health technology assessment in surgical interventions requires an initial evaluation of the safety and stability of new interventions followed by randomised trials [15]. The initial evaluative evidence alone is not sufficient to assess the clinical effectiveness of LUNA for which randomised research remains the gold standard.

Evidence from the medical literature: In the past, pelvic denervation has been proposed for treatment of chronic pelvic pain [13] but it has not been widely adopted due to the need for open invasive surgery. However, recent developments in minimal access surgery make LUNA a practicable treatment option. Our systematic review [16] has shown that the currently available research evidence on LUNA is inconclusive. Therefore further research is required to generate effectiveness evidence in the form of a high quality randomised controlled trial. This need is also supported by the recent Cochrane Reviews $[17,18]$ that recommend rigorous research to assess surgical interventions in chronic pelvic pain.

\section{Professional consensus}

We conducted a survey of UK gynaecologists associated with the British Society of Gynaecological Endoscopy in 1998 to determine the extent to which LUNA was being used in practice [19]. Our survey indicated that despite the lack of definitive evidence, many gynaecologists familiar with the technique were using LUNA as a therapeutic option. Of the 247 respondents, 108 (44\%) offered LUNA to patients: $78 \%$ for chronic pelvic pain, $66 \%$ endometriosis and $18 \%$ for dyspareunia/other reasons. Crucially, this survey indicated that 93 of 108 (86\%) gynaecologists currently performing LUNA were willing to recruit patients in a randomised trial of LUNA. In this situation equipoise applies (i.e. the technique has been introduced without definite evidence but opinion regarding its use is not yet solidified) making the need for a trial even more urgent.

\section{Pilot study}

We have undertaken a pilot of the LUNA trial with the objective of assessing its feasibility. It has shown acceptability to patients. It has also established trial management procedures, piloted questionnaires, measured compliance and standardised operating procedures. A confidential 
interim analysis was recently reviewed by an independent data monitoring committee when the first 60 patients had completed 6 months follow-up. The committee recommend that a larger study is needed for adequate statistical power in the trial to evaluate LUNA reliably.

\section{Research Proposal Objectives}

1. To test the hypothesis that in women with chronic pelvic pain in whom diagnostic laparoscopy reveals either no pathology or mild endometriosis (AFS score $\leq 5$ ) LUNA alleviates pain and improves life quality at 12 months (principal objective).

2. To test the hypothesis that response to LUNA differs according to the site and cause of the pain by two secondary analyses: (i) Women with central pain, (ii) women with no visible pathology.

3. To explore the variation in LUNA's effectiveness and side effects at different periods of follow-up $(3,6$, months and $1,2,3,5$ and 10 years).

\section{Design and setting}

A multi-centre, prospective, randomised-controlled-trial involving centres in the UK and elsewhere will be carried out with single blind assessment of outcomes in eligible consenting patients randomised at diagnostic laparoscopy to LUNA (experimental group) or to no pelvic denervation (control group). Postal questionnaires including visual analogue scale for pain (primary outcome), an index of sexual satisfaction and the EuroQol 5D-EQ instrument (secondary outcomes) will be administered at 3, 6 and 12 months. The primary assessment of the effectiveness of LUNA will be from comparison of outcomes at the oneyear follow-up, although the medium-term and long-term risks and benefits of LUNA will also be evaluated postal questionnaires to the women at 2, 3, 5 and 10 years after laparoscopy.

\section{Eligibility}

All new patients presenting to the Gynaecology outpatient clinic with pelvic pain (cyclical or noncyclical) and/or dyspareunia, and requiring diagnostic laparoscopy for evaluation of these conditions, will be invited to participate (Protocol, patient information leaflet and the relevant forms and questionnaire available on the LUNA trial website)[20]. They will be provided with information leaflet-Appendix A1 [see Additional file 1]. When they consent to participate (for consent form see Appendix A2additional file 1$)$, they are registered prior to randomisation (Appendix B- additional file 1) and an On study form is filled (Appendix C-see additional file 1)

\section{Inclusion criteria}

- Pelvic pain of longer than 6-month duration.

- Pain located within the true pelvis or between and below the anterior iliac crests.

- Associated functional disability.

- Lack of response to medical treatment.

- Diagnostic laparoscopy planned.

\section{Exclusion criteria}

- Previous LUNA.

- Mild, moderate and severe endometriosis (American Fertility Society score $>5$ ).

- Previous surgery for endometriosis.

- Previous surgery for pelvic inflammatory disease.

- Previous hysterectomy.

- Adnaexal pathology.

\section{Interventions}

Diagnostic laparoscopy plus uterosacral nerve ablation (experimental group) or laparoscopy without pelvic denervation (control group).

Laparoscopic uterosacral nerve ablation will be carried out in a uniform manner by named surgeons in each of the participating centres following a common protocol as described in the standard surgical text [21]. Routine preparation will be made for a diagnostic laparoscopy with the patient under general anaesthesia. Following pneumoperitoneum, a laparoscope will be used to visualise the pelvis. Before embarking on operative laparoscopy an anatomical pelvic assessment will be performed to identify pelvic structures and pathology. At this stage patients with pathology outlined in the exclusion criteria will be excluded. It is expected that around 30\% of women will be unsuitable for LUNA at operation and will be 'registered only' cases. Eligible patients will be randomised by a telephone call to the Birmingham University Clinical Trials Unit.

Clear identification of the uterosacral ligaments is a prerequisite to treatment with lasers or electro-diathermy. The posterior leaf of the broad ligament will be carefully inspected to identify the course of the ureters, which on rare occasions could be particularly close to the uterosacral ligaments. Care will also be taken to note thin walled pelvic veins, which often lie lateral to the uterosacral 
ligaments. If accidentally punctured, they may cause troublesome bleeding requiring further endoscopic endocoagulation. The uterosacral ligaments will be identified by manipulation of the uterus in the right and left lateral planes. The ligaments will then be ablated with laser or micropoint electro-diathermy or endocoagulation depending upon the surgeons' preference. The ablation will start as close to the posterior aspect of the cervix as possible and continue for a minimum of $1 \mathrm{~cm}$ posterolaterally on either side. The aim of the procedure is to destroy the sensory nerve fibres and the secondary ganglia as they leave the uterus and come to lie within the uterosacral ligaments.

The safe conduct of operative laparoscopy for LUNA requires the use of two ports, one for delivery of the energy source (laser or diathermy) and another for manipulation. These are in addition to the umbilical port used for the laparoscope itself. In contrast, diagnostic laparoscopy in women with no pathology requires only one port in addition to the umbilical laparoscopic port. This difference in number of ports has potential for introducing bias by compromising patient blinding to group allocation. A sham incision (see Blinding and Ethical Considerations) in the control group is used to overcome this problem.

\section{Randomisation}

Consenting eligible patients will be randomised to diagnostic laparoscopy plus uterosacral nerve ablation (experimental group) or to no pelvic denervation at the time of diagnostic laparoscopy (control group). Important issues related to randomisation are considered below:

\section{Allocation Sequence}

The subjects will be allocated to groups using a chance procedure, blocking and stratification [22]. Stratified block randomisation will be employed to ensure that there will be nearly equal numbers of patients in the two groups within the prognostic subgroups, even if the study ends prematurely. Variable block size will be used to avoid any possibility of foreknowledge. Stratified allocation is used so that chance imbalances in the stratification variable do not have an effect on the outcome. Since response to treatment may depend on the surgeons' technique for laparoscopic uterosacral nerve ablation, analyses will be retrospectively stratified according to the surgeons participating in the trial.

\section{Intraoperative randomisation}

Allocation concealment is a crucial factor in avoiding bias in randomised trials [23]. Although it is not possible to blind the surgeon, it is essential to keep the surgeon blind to the group allocation until after the irrevocable decision to enter the woman into the trial has been made. So, in LUNA, treatment allocation will be issued at diagnostic laparoscopy, after the surgeon has inspected the pelvis and ensured that the patient fulfils all of the inclusion criteria and she does not have any of the exclusion criteria. Women may be randomised or registered into the study by telephoning the toll free Randomisation Line on 0800 $9530274(+441216872319$ from outside the UK) or by Internet randomisation at http://www.luna.bham.ac.uk and clicking on the randomisation button. Passwords for Internet randomisation will only be allocated to centres with ethical approval.

Following surgery, the surgeon fills in operation details on a post-surgery form (Appendix D- see additional file 1)

\section{Blinding}

In the LUNA study, patients will be kept blind to their treatment allocation until the follow-up in the trial is complete. Patients may also show a placebo effect if they know they have received the active treatment. The magnitude of placebo effects should not be underestimated. There is clear evidence that inadequacies of blinding in randomisation lead to exaggeration of treatment effect in randomised trials [24].

However, there is a potential problem in the maintenance of blinding in the LUNA trial. As mentioned earlier, patients allocated to have LUNA will have the standard operative laparoscopy with three ports (one $10 \mathrm{~mm}$ umbilical port and two $5 \mathrm{~mm}$ lateral ports), whereas patients allocated to the control group under normal circumstances would have standard diagnostic laparoscopy with two ports (one $10 \mathrm{~mm}$ umbilical port and one $5 \mathrm{~mm}$ lateral or midline port). By noting the different number of incisions some patients might become aware of their group allocation and this might alter their response. In order to maintain patient blinding, a sham $5 \mathrm{~mm}$ skin incision is made superficially in a lateral port site. This approach in avoiding bias due to lack of blinding has been used in a previous trial of laparoscopic nerve ablation [25] and has also received ethical approval in this trial.

A second purpose of blinding is to prevent differences in other aspects of patient management introducing biases affecting the results. The patient's GP will therefore be kept blind to treatment allocation. Double blinding is not possible in LUNA, however, as the surgeons performing the surgical intervention on the patients will be aware of the group allocation. However, the likelihood that this will lead to bias in outcome assessment is low as the patient outcome assessments in this study will be conducted by self-administered questionnaires, avoiding any possible bias from surgeons' knowledge of group allocation. 


\section{Endpoints \\ Primary Outcome measure: Visual analogue scale for assessment of pain}

Pain is difficult to measure, partly because it is accompanied by other sensations and partly because the reaction component affects the judgement of the pain regardless of the intensity of the stimulus. A measure of pain is nevertheless essential to the outcome of this clinical trial. Visual analogue scales (VAS) originally devised as measures of well being, [26] have been successfully adapted to measure pain $[27,28]$. This technique involves use of a $10 \mathrm{~cm}$ line on a piece of paper representing a continuum of the patients' opinion of the degree of pain. It is explained to the patient that the one extreme of the line represents "no pain at all" while the other represents "as much pain as she can possibly imagine". The subject rates the degree of pain by placing a mark on the line and scale values are obtained by measuring the distance from zero to that mark. Visual analogue scales (VAS) in the assessment of pain have been established to be reproducible and accurate [28]. VAS has commonly been used in measurement of chronic pain [29]. All the studies of LUNA included in systematic reviews so far have used this measure, or a variation on it, for assessing outcome. Individual pain scores have sufficient psychometric strengths to be used in chronic pain research involving group comparison designs[30].

\section{Secondary Outcome measures}

Assessment of sexual function

Sexual function is an important aspect of life quality in patients with pelvic pain. Pain itself is an anti-aphrodisiac, and together with discomfort and altered self image, it impacts upon sexual function [31]. Its assessment in an objective manner is therefore an important part of the LUNA trial. There are several sexual function instruments, with high levels of reliability, validity and responsiveness, which yield comparable results across occasions and individuals, making them suitable for monitoring therapeutic progress in randomised research [31].

The Sexual Activity Questionnaire (SAQ)[32] will replace the Brief Index of Sexual Satisfaction (ISS) [33] for the assessment sexual function. The outcome measure has been changed because of poor acceptability and compliance with the ISS. The SAQ has excellent internal consistency and test retest reliability. It also has excellent concurrent and construct validity and has been shown to be acceptable to women in other clinical trials [34] In the questionnaire it will be clearly stated that the measure of sexual function covers material that is sensitive and personal. Participants will be reassured that their responses will be kept completely confidential and that if they do not wish to answer any questions, they will be allowed to leave the questionnaire blank.
Health-related quality of life Instrument

Health-related quality of life (HRQL) instruments are becoming powerful tools for outcome assessments in randomised trials. Quality of life instruments assess aspects of patient's health status usually not grasped by conventional clinical indices; hence, they can be applied as complementary assessments together with VAS and ISS. Quality of life has to be defined clearly and patient's perception of normal performance serves a pivotal role in this context. HRQL instruments are administered with questionnaires assessing a number of different domains, i.e. areas of behaviour or experience that the instrument is attempting to measure [35].

Resource usage outcome measures

LUNA is a quick, safe and inexpensive procedure for women already undergoing diagnostic laparoscopy. Our hypothesis is about a clinically important effect without an excess of complications. If the hypothesis is confirmed, then any benefits will essentially be "dominant" outweighing the relatively small costs of intervention. Therefore, we do not plan a formal economic evaluation at this stage. However, data on health resource use will be collected partly as efficacy outcome measures (i.e. less need for medical care for pelvic pain indicates greater efficacy), and partly to allow an economic evaluation to be carried out, should significant complications occur. Other measures will include analgesic use, consultations at general practice and hospital, and time off work. Again these are both economic outcomes and indicators of residual pain.

\section{Follow-up}

Postal Questionnaires to assess pain and sexual function will be administered at enrolment in the trial and then at 3, 6, 12, 24 and 36 months after laparoscopy. The outcomes at 12 months will be used to address the primary research question. This time interval is chosen because laparoscopy alone has a placebo effect for up to 3-6 months in some patients [36,37]. The 24 and 36-month follow up will be used to monitor medium-term effects of the intervention. Existing participants in the trial who consented to 3 years of follow-up will be asked to consent to long-term follow-up (10 years) once they have reached the 3 year follow up time-point. Participants randomised after June 2003 are asked to consent to 10 years of followup at entry for long term follow up.

\section{Ineligible Patients - follow-up only}

Women who are ineligible for the LUNA trial because of moderate to severe endometriosis, significant adhesions, significant pelvic inflammatory disease, other significant pathology or those for whom LUNA is not technically feasible should be registered with the Trial Office for followup only. The woman should be told that she was not eligible for the trial randomisation, and the reasons why, 
and asked if she would agree to complete the follow-up questionnaires at 6 and 12 months.

This non-random cohort will provide comparative data on the natural history of patients with chronic pelvic pain with significant pathology.

\section{Side-effects quantification}

The centres have been advised to fill an " adverse event form" in case of immediate and delayed complications if any are associated with the procedure.

\section{Sample Size and Power Considerations}

The sample size for this trial has been estimated using the hypothesis that LUNA will alleviate pain symptoms (i.e. reduce pain scores on a VAS) more often than no intervention at one-year following diagnostic laparoscopy. Cohen describes 'effect sizes' of 0.2 and 0.5 standard deviations (SD) as 'small' and 'medium' [38]. Interim analyses of the pilot study indicate that the SD of the difference in change in VAS scales between LUNA and no pelvic denervation groups will be about 4.0. This corresponds to small and medium effect sizes on VAS of 0.8 and 2.0 respectively and is consistent with other studies of chronic pelvic pain, where clinically important symptom alleviation has been defined as a reduction in pain score of 2 or more [39]. To confirm or refute a small to medium effect of LUNA ( 0.3 SD difference), based on $\dot{\alpha}=0.05$ and $\beta=0.2(80 \%$ power), 175 patients in each group (i.e. 350 patients in total) will be required. Considering a $20 \%$ loss to followup, the sample size is inflated to 210 patients in each group (i.e. 420 patients in total).

\section{Stratification Variables}

Randomisation will be conducted using minimisation, stratified by the four variables:

a. Presence or absence of some minimal pathology (minimal endometriosis \pm ablation; adhesions requiring adhesiolysis only; minimal pelvic inflammatory disease)

b. Site of pain (presence of central pain or not)

c. Parity of the woman (nulliparous or parous)

d. Whether the woman is sexually active or not

The first two variables form the prespecified subgroup analyses, and the other two variables are included as having impact on dysmenorrhoea and dyspareunia respectively.

\section{Data Analysis}

Type of analysis

Intention to treat
The main analysis to address the principal research questions will be conducted using the one-year follow-up data. The mean differences in VAS pain scores; sexual satisfaction and life quality scores in the two groups will be compared using a two-sample t-test. The rates of women with clinically significant (2 VAS point) alleviation of pain symptoms will also be compared producing a relative risk estimate with 95\% confidence intervals (Mantel-Haenzel test). Baseline characteristics of the patients enrolled in the two groups will be compared to ensure that randomisation has produced comparable groups of patients. The use of additional treatment (co-intervention) for pelvic pain following LUNA or no pelvic denervation will be assessed for any systematic difference between the two groups.

Subgroup analyses are limited by statistical power and can produce spurious results particularly if many are undertaken. Our literature review [16] and consultation with gynaecologists [19] suggests that the effectiveness of LUNA may be greater for central compared to non-central pain and if there is no associated pathology (i.e. no endometriosis). Therefore, we have chosen to limit secondary analyses to these subgroups only. The LUNA trial is powered to detect a small to medium overall difference and if a larger treatment benefit is found then other subgroup analyses will be undertaken, appropriately cautiously. The LUNA trial is powered overall at $80 \%$ to detect a 0.3 SD difference in effect. Our pilot study shows that $60 \%$ patients have mainly central pain and $70 \%$ have no pathology. Hence, in the subgroup with central pain the power will be $80 \%$ to detect a 0.4 SD treatment effect. In the subgroup with no pathology the power will be $80 \%$ to detect a $0.35 \mathrm{SD}$ treatment effect.

\section{Frequency of analyses}

Interim analysis at completion of 6 months follow-up of the first 60 patients has already been completed as pilot study.

Bi-annual analyses of recruitment, compliance and loss to follow-up are being carried out for LUNA Trial Management Committee.

An annual interim analysis of effectiveness is done for confidential review by independent Data Monitoring Committee to determine whether the principal question has been answered and to monitor adverse events.

Main analyses of effectiveness of LUNA will be done at completion of one year follow up of the total study sample. Additional analysis of long-term effects will be done at two and three-year follow-up points. 


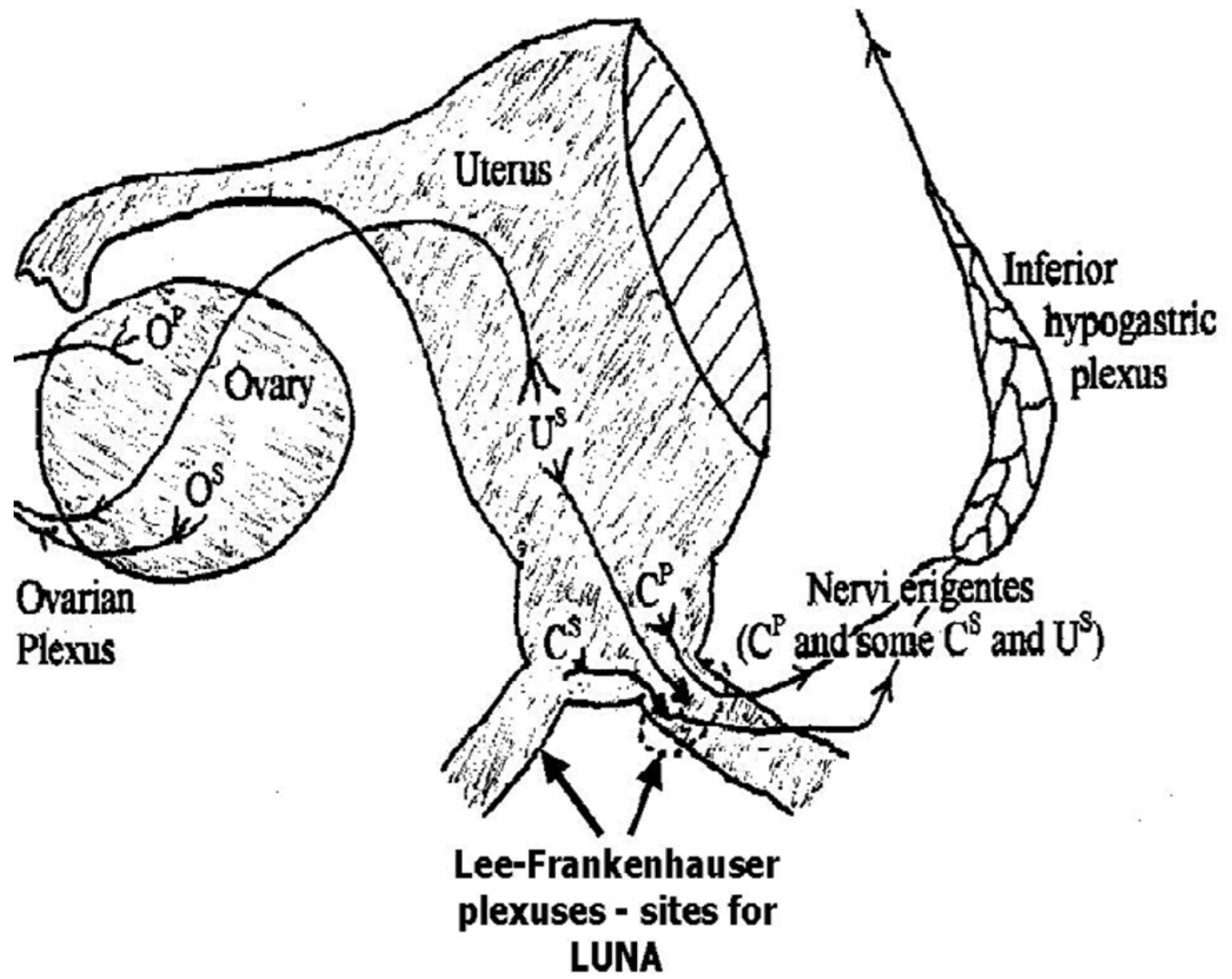

Figure I

Pelvic sensory nerve pathways and site for luna

\section{Ethical considerations}

When there is uncertainty about the appropriate therapy, scientific clinical trials are the best scientifically ethical way to resolve uncertainty and thereby benefit both the individual patients and all others concerned in their care [40]. The need for a "sham" incision in this trial is the main ethical issue and it is required because without it the patients cannot be kept blinded. The purpose of blinding is to prevent various biases from affecting the results. The need for blinding in surgical trials has been emphasised in the medical literature [41] and there is empirical evidence that inadequacies of blinding in randomisation lead to exaggeration of therapeutic efficacy in randomised trials [42]. Blinding of patients in surgical trials is clearly indicated when the intervention primarily treats symptoms and when the outcomes are based on patients' own assessment. LUNA is an intervention for treating chronic pelvic pain (a diagnosis based on symptoms) and the outcome assessment is based on patients' responses on a visual analogue pain scale and a quality of life instruments. Hence, the use of a "sham" incision is justified if bias is to be avoided in the LUNA trial and this approach has been used in a previous trial of LUNA [43]. Ethical approval for the LUNA trial procedures has already been obtained from the Multicentre Research Ethics Committee (MREC).

\section{Conduct of the Study and Data Management}

The trial will be managed from the Birmingham Clinical Trials Unit. Each investigating centre will carry out the study in accordance with the study protocol and to the MRC guidelines on Good Clinical Practice in Clinical Research (1998). Patients will be invited to participate if they fulfil all the inclusion criteria and do not have any exclusion criteria (See Appendix B Eligibility Checklist and Randomisation Form). They will be provided with a laparoscopy and LUNA trial information leaflet (Appen- 


\begin{tabular}{|c|c|c|c|c|c|c|c|c|c|}
\hline & \multirow[t]{2}{*}{ Clinic } & \multirow[t]{2}{*}{ Theatre } & \multicolumn{7}{|c|}{ Follow up (months/ years) } \\
\hline & & & 3 & 6 & 12 & 2 & 3 & 5 & 10 \\
\hline Patient information and consent (App A) & $x$ & & & & & & & & \\
\hline Eligibility Checklist and Randomisation form (App B) & $x$ & $x$ & & & & & & & \\
\hline Enrolment Questionnaire (Appendix Cl) & $x$ & & & & & & & & \\
\hline Surgery Form (Appendix D) & & $\mathrm{x}$ & & & & & & & \\
\hline Letter to GP (Appendix E) & $x$ & & & & & & & & \\
\hline Follow-up Questionnaire (Appendix C2, C3) & & & $x$ & $x$ & $x$ & $x$ & $x$ & $x$ & $x$ \\
\hline
\end{tabular}

dix A1) and signed consent obtained prior to laparoscopy (Appendix A2). Consenting patients should be asked to complete the Enrolment Questionnaire and On Study Form. The final decision to enrol patient in the trial will depend on the findings at laparoscopy when the surgeon will perform LUNA or not after determining eligibility as shown in Eligibility Checklist and Randomisation Form (see Appendix B). At the end of the procedure the surgeon will complete the Post Surgery Form (Appendix D). All the three forms for each patient enrolled will be photocopied to keep a record at the participating centre and the originals will be sent to the Birmingham Clinical Trials Unit, which will act as the co-ordinating centre. At 3, 6 and 12 months after enrolment, the Follow-up Questionnaire will be mailed to the patients with a pre-paid selfaddressed envelope. Recruitment is expected to take 12 months and follow up for the main endpoints another 12 months. At completion of the main study further Followup questionnaires will be mailed out at 24 and 36 months.

Finishing date

September 2005; expected reporting date: December 2006

\section{List of abbreviations used}

AFS- American Fertility Society

5D EQ- 5 dimensional European Quality of Life Questionnaire

GP- general practitioner

LUNA- laparoscopic uterosacral nerve ablation

RR- relative risk

95\% CI- 95\% confidence intervals

VAS- visual analogue scale

HSG-health service guidelines
SAQ- Sexual Activity Questionnaire

HRQL- Health-related quality of life

\section{Competing interests}

None declared.

\section{Authors' contributions}

The LUNA Trial Collaboration consists of a management group and a collaborative group.

The management group consists of Pallavi Latthe (PL), Khalid S Khan (KSK) Janesh K Gupta (JKG) from Department of Obstetrics and Gynaecology, Jane Daniels (JD), Robert K Hills (RKH) and Richard Gray (RG) from Birmingham Clinical Trials Unit, and Richard Lilford (RJL) from Department of Public Health and Epidemiology, all from University of Birmingham, UK. KSK and RG were involved in developing the original idea of the trial. KSK, JKG, RJL and RG were co-applicants on the successful funding proposal and are responsible for the overall direction of the project. PL, JD and RKH have contributed to adaptations from the original design. PL co-ordinates clinical work within various study centres and recruits patients along with other collaborators. RG and RKH provide statistical advice. All of the above authors are members of the trial management group and have approved the final manuscript.

The collaborative group consists of clinicians and centres that recruit patients in this trial. At the time of submitting this protocol, the following clinicians (centres) have recruited patients: P Chein (Ninewells Hospital, Dundee, UK); K Chin (Staffordshire General Hospital, UK); M O'Connor, A Baxter, T Farrell (Royal Hallamshire Hospital, Sheffield, UK); LS Dwarakanath (City Hospital, Birmingham, UK); S Irani (Birmingham Heartlands and Solihull Hospital NHS Trust, UK); KSK, PL, JKG, C Mann, TJ Clarke (Birmingham Women's Hospital, Birmingham, UK); V Kay (Forth Park Hospital, Kirkcaldy, UK); D Phillips (Perth Royal Infirmary, UK); JS Samra, C Cox (New 


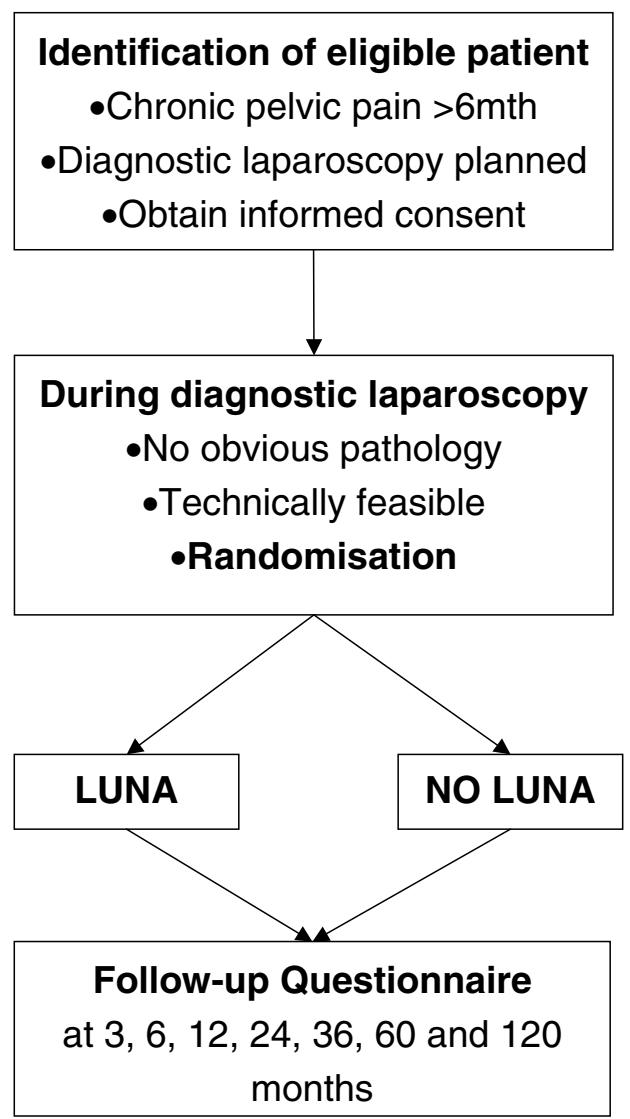

Figure 2

The laparoscopic uterosacral nerve ablation (luna) trial schema

Cross Hospital, Wolverhampton, UK); S Keay (Walsgrave Hospital, Coventry, UK); E Shaxted (Northampton General Hospital, UK) and J Thornton (City Hospital, Nottingham, UK) The following centres have obtained ethics approval and await recruitment: A Ali (Darlington Memorial Hospital, UK); C Chandler (Billinge Hospital, Wigan, UK); E Downes (Chasefarm Hospital, Enfield, UK); A Hextall (St Albans City Hospital, UK);J Kabukoba (Sandwell Hospital, West Bromwich, UK); M Katesmark (Epsom General Hospital, UK); P. Kirwan (Leicester General Hospital, UK); K Louden (Royal Hampshire Hospital, UK);J Moohan (Altnagelvin Area Hospital, UK); S Najja (Queen's Park Hospital, Blackburn, UK); K Olah (Warwickshire General Hospital, UK); M Padwick (Watford General Hospital, UK); P Owen (Stobhill Hospital, Glasgow, UK) and A Tapp (Royal Shrewsbury Hospital, UK).

\section{Additional material}

\section{Additional File 1}

Appendix A1: Patient information leaflet. Appendix A2: Consent form. Appendix B: Registration and randomisation form. Appendix C: On study form. Appendix D: Post surgery form.

Click here for file

[http://www.biomedcentral.com/content/supplementary/14726874-3-6-S1.doc]

\section{Acknowledgements}

Funding: WellBeing, UK (Charity grant no. CF/37I)

Data Monitoring Committee: P Brocklehurst, D Braunholtz and JA Jordan

\section{References}

I. M Morris, D O'Niell: Out patient Gynaecology. BMJ I958, 2: 1038.

2. Howard FM: The role of laparoscopy in chronic pelvic pain: promise and pitfalls. Obstet Gynecol Surv 1993, 48:357-387.

3. Stones RW: Chronic pelvic pain. Personal Assessment in Continuing Education. 97/0 I th edition. London, Royal College of Obstetricians and Gynaecologists; 1997.

4. Sundell G, Milsom I, Andersch B: Factors influencing the prevalence and severity of dysmenorrhoea in young women. $\mathrm{Br}$ J Obstet Gynaecol 1990, 97:588-594.

5. Davies L, Ganger K, Drummond M, Saunders D, Beard R: The economic burden of intractable gynaecological pain. J Obstet Gynecol 1992, I 2:46-54

6. Zondervan KT, Yudkin PL, Vessey MP, Dawes MG, Barlow DH, Kennedy SH: Prevalence and incidence of chronic pelvic pain in primary care: evidence from a national general practice database. Br J Obstet Gynaecol 1999, I 06: I | 49- I I 55.

7. Vercellini P, Fedele L, Arcaini L, Bianchi S, Rognoni MT, Candiani GB: Laparoscopy in the diagnosis of chronic pelvic pain in adolescent women. J Reprod Med 1989, 34:827-830.

8. MR Henzl: Dysmenorrhoea; achievements and challenge. Sex Med Today 1985, 9:8-12.

9. Porpora MG, Gomel V: The role of laparoscopy in the management of pelvic pain in women of reproductive age. Fertil Steril 1997, 68:765-779.

10. G Frankenhauser: Die Bewegungenerven der Gebarmutter. Z Med Nat Wiss 1864, I:35.

II. Latarjet A Roget P: Le plexus hypogastrique chez la femme. Gynecologie et Obstetrique 1922, 6:225.

12. RM Campbell: Anatomy and physiology of sacrouterine ligaments. Am J Obstet Gynecol 1950, 59:I.

13. JB Doyle: Paracervical uterine denervation by transection of the cervical plexus for the relief of dysmenorrhoea. $\mathrm{Am} J$ Obstet Gynecol 1955, 70: I.

14. Doyle JB Des Rosiers J): Paracervical uterine denervation for the relief of pelvic pain. Clinical Obstet Gynecol 1963, 6:742-753.

15. A Cushcieri: Minimal Access Therapy: scope, evaluation and future direction. Health Bull 1996, 54:5 |4-52 I.

16. Khan KS, Khan SF, Nwosu CR, Dwarakanath LS, Chien PFW: Laparoscopic uterosacral nerve ablation in chronic pelvic pain: An overview. Gynaecological Endoscopy 1999, 8:257-265.

17. Wilson ML, Farquhar CM, Sinclair OJ, Johnson NP: Surgical interruption of pelvic nerve pathways for primary and secondary dysmenorrhoea. [Review] [6 refs]. Cochrane Database Syst Rev 2000:CD001896.

18. Stones RW, Mountfield ]: Interventions for treating chronic pelvic pain in women. [update of Cochrane Database Syst Rev. 2000;(2):CD000387; I07967I3.]. [Review] [I 5 refs]. Cochrane Database Syst Rev 2000:CD000387.

19. Daniels J, Gray R, Khan K, Gupta J: Laparoscopic uterine nerve ablation: A survey of gynaecological practice in the UK. Gynaecol Endosc 2000, 9:157-I59.

20. Unit University of Birmingham Clinical Trials: The LUNA Trial. http://www luna bham ac uk 2003. 
21. Sutton $C$ Whitelaw $N$ : Laparoscopic uterine nerve ablation for intractable dysmenorrhoea. Endoscopic surgery for Gynaecologists Edited by: Sutton CDiamond MP. London, WB Saunders; 1993:159-163.

22. Zelen M: The randomization and stratification of patients to clinical trials. J Chronic Dis 1974, 27:365-375.

23. Schulz KF, Chalmers I, Hayes RJ, Altman DG: Empirical evidence of bias. Dimensions of methodological quality associated with estimates of treatment effects in controlled trials. JAMA 1995, 273:408-4I2.

24. Schulz KF, Chalmers I, Hayes RJ, Altman DG: Empirical evidence of bias. Dimensions of methodological quality associated with estimates of treatment effects in controlled trials. JAMA 1995, 273:408-4I2.

25. Sutton CJ, Ewen SP, Whitelaw N, Haines P: Prospective, randomized, double-blind, controlled trial of laser laparoscopy in the treatment of pelvic pain associated with minimal, mild, and moderate endometriosis. Fertil Steril 1994, 62:696-700.

26. Clarke PRF Spear FG: Reliability and sensitivity in the self assessment of wellbeing. Bull Br Psychol soc 1964, 17:55.

27. Bond MR, Pilowsky I: Subjective assessment of pain and its relationship to the administration of analgesics in patients with advanced cancer. J Psychosom Res 1966, 10:203-208.

28. Revill SI, Robinson JO, Rosen M, Hogg MI: The reliability of a linear analogue for evaluating pain. Anaesthesia 1976, 31:1191-1198.

29. Carlsson AM: Assessment of chronic pain. I. Aspects of the reliability and validity of the visual analogue scale. Pain 1983, 16:87-101.

30. Jensen MP, Turner JA, Romano JM, Fisher LD: Comparative reliability and validity of chronic pain intensity measures. Pain 1999, 83:157-162

31. D Jehu: Sexual dysfunction- a behavioural approach to causation, assessment, and treatment Toronto, John Wiley and sons; 1979.

32. Thirlaway K, Fallowfield L, Cuzick J: The Sexual Activity Questionnaire: a measure of women's sexual functioning 2 . Qual Life Res 1996, 5:8I-90.

33. WW Hudson: Index of sexual satisfaction. The clinical measurement package: a field manual 1982th edition. Chicago: Dorsey Press; | 982:190-191.

34. Stead ML, Crocombe WD, Fallowfield LJ, Selby P, Perren TJ, Garry R, Brown JM: Sexual activity questionnaires in clinical trials: acceptability to patients with gynaecological disorders I I. $\mathrm{Br}$ J Obstet Gynaecol 1999, 106:50-54.

35. Guyatt GH, Feeny DH, Patrick DL: Measuring health-related quality of life. Ann Intern Med I 993, I I 8:622-629.

36. Fedele L, Marchini M, Acaia B, Garagiola U, Tiengo M: Dynamics and significance of placebo response in primary dysmenorrhea. Pain 1989, 36:43-47.

37. Baker PN, Symonds EM: The resolution of chronic pelvic pain after normal laparoscopy findings. Am J Obstet Gynecol 1992, 166:835-836.

38. J Cohen: Statistical power analysis for the behavioural sciences. 1977.

39. Zullo F, Pellicano M, DeStefano R: Efficacy of Laparoscopic Pelvic Denervation in Central-type Chronic Pelvic Pain: A multicentre study. J Gynecol Surg 1996, I 2:35-40.

40. Chalmers T: The clinical trial. Milbank Memorial Fund Quarterly 1981, 59:339-342.

41. Johnson AG, Dixon JM: Removing bias in surgical trials. $B M$ |997, 3 | 4:9|6-9|7.

42. Schulz KF, Chalmers I, Hayes RJ, Altman DG: Empirical evidence of bias. Dimensions of methodological quality associated with estimates of treatment effects in controlled trials. JAMA |995, 273:408-4|2.

43. Sutton CJ, Ewen SP, Whitelaw N, Haines P: Prospective, randomized, doubleblind, controlled trial of laser laparoscopy in the treatment of pelvic pain associated with minimal, mild, and moderate endometriosis. Fertil Steril 1994, 62:696-700.

\section{Pre-publication history}

The pre-publication history for this paper can be accessed here:

http://www.biomedcentral.com/1472-6874/3/6/prepub
Publish with Bio Med Central and every scientist can read your work free of charge

"BioMed Central will be the most significant development for disseminating the results of biomedical research in our lifetime. "

Sir Paul Nurse, Cancer Research UK

Your research papers will be:

- available free of charge to the entire biomedical community

- peer reviewed and published immediately upon acceptance

- cited in PubMed and archived on PubMed Central

- yours - you keep the copyright 